Retos, $\mathrm{n}^{\circ} 13$, vol.VII, 2017

\title{
La interacción personal y su efecto en la decisión de compra
}

\section{Personal interaction and its effect on the purchase decision}

\author{
Raúl Álvarez Guale \\ Universidad Politécnica Salesiana. Ecuador \\ ralvarezg@ups.edu.ec \\ orcid.org/0000-0002-3173-4926 \\ Fabián Villacrés Beltrán \\ Universidad Politécnica Salesiana. Ecuador \\ fvillacres@ups.edu.ec \\ orcid.org/0000-0003-4779-5608
}

\section{Resumen}

La presente investigación trata de responder el cuestionamiento acerca del nivel de relación que existe entre la interacción personal, basada en la calidad del servicio, y la decisión de compra de los consumidores en los supermercados de la ciudad de Guayaquil, planteándose como objetivo general la determinación de la relación de ambas variables. Además se plantean otros objetivos que buscan determinar la influencia de esta interacción personal con el proceso y comportamiento de compra de los consumidores. Mediante la aplicación de una encuesta de 30 preguntas y de un análisis correlacional-explicativo se obtienen coeficientes de Tau b de Kendall en las variables y dimensiones de estudio analizados en las 4 cadenas de comisariatos más importantes por volumen de ventas de la ciudad de Guayaquil (Ecuador), siendo éstas: Almacenes TIA, Mi Comisariato, Aki y Gran AKI, y las cadenas de Supermaxi-Megamaxi. Se muestran influencias importantes que describen una relación entre la interacción personal con la decisión de compra y en el contexto del proceso y comportamiento de compra de los consumidores. Las conclusiones más relevantes encontradas en este estudio hacen referencia en la aceptación de la las hipótesis planteadas, ya que en cada uno de los casos estudiados, los valores $p$ de la significancia bilateral son inferiores al $5 \%$.

\begin{abstract}
This research attempts to answer the question about the level of relationship between personal interaction, based on the quality of service, and the purchase decision of consumers in supermarkets in the city of Guayaquil, raising overall objective determination of the relation of both variables. In addition other objectives that seek to determine the influence of this personal interaction with the process and buying behavior of consumers arise. By applying a 30-question survey and a correlational-explanatory analysis, we obtain Kendall's Tau b coefficients in the variables and study dimensions analyzed in the 4 most important comisariat chains by volume of sales in the city of Guayaquil ( Ecuador), those are: Almacenes TIA, Mi Comisariato, AKi y Gran AKI, and the supermarket chains Supermaxi-Megamaxi. Important influences that describe a relationship between personal interaction with the purchase decision are shown and in the context of the process and buying behavior of consumers. The most relevant conclusions found in this study refer to the acceptance of the hypotheses, since in each of the cases studied, the $p$ values of bilateral significance are less than $5 \%$.
\end{abstract}

\section{Palabras clave | keywords}

Servicios, consumidor, comportamiento, comportamiento del consumidor, calidad, interacción. Services, consumers, behavior, consumer behavior, quality, interaction. 


\section{Introducción}

Según el censo Económico 2010, realizado y presentado por el Instituto de Estadística y Censo del Ecuador en el año 2011, la actividad económica del comercio al por mayor y al por menor; reparación de vehículos automotores y motocicletas fue una de las actividades con mayor incidencia de participación en el mercado con $29.7 \%$ en personal ocupado, $16.1 \%$ en gasto por remuneración, $20.9 \%$ en obtener financiamiento, $30.31 \%$ en uso del Internet, $18.89 \%$ en gastos de electricidad y un $41.35 \%$ en totales de ingresos. A esta actividad le sigue la actividad económica de industrias Manufactureras con una incidencia de participación en el mercado con $12.9 \%$ en personal ocupado, $11.5 \%$ en gasto por remuneración, $14.9 \%$ en obtener financiamiento, $9.7 \%$ en uso del Internet, $46.7 \%$ en gastos de electricidad y un $27.7 \%$ en totales de ingresos. Ambas actividades se encuentran muy correlacionadas con los ingresos y gastos de energía, e inclusive eliminando toda correlación espuria existente (Álvarez-Guale, 2011).

En la Universidad Politécnica Salesiana del Ecuador se han realizado varios estudios enfocados a la elaboración de Modelos para evaluar la calidad del servicio en varias actividades económicas. En el trabajo «Creación y aplicación de un modelo de evaluación de la calidad del servicio orientado a la reparación de vehículos automotores y motocicletas en la ciudad de Guayaquil, aplicada a 6 vulcanizadoras de la Parroquia Ximena» (Alvarado-Contreras \& Arteaga-Mendoza, 2013), el resultado presenta un modelo bidimensional donde se realizan 42 preguntas cruzadas.

Este modelo propuesto reduce de 119 preguntas iniciales a 42, siendo representativas al $98.8 \%$ del total de preguntas iniciales, es decir, que las 42 preguntas representan significativamente el $98.8 \%$ de las 119 preguntas. Con la aplicación del modelo de las 42 preguntas, la actividad económica tuvo una valoración de la calidad del servicio de 6.33 sobre 10 puntos.

Otra propuesta basada en los mismos parámetros, para determinar la calidad del servicio de distribución y venta de combustibles, según corresponda a la actividad económica venta al por menor de combustibles para automotores en la ciudad de Guayaquil es la de CampoverdeGuerra \& Pérez Beltrán (2013), en la que se propone un modelo para evaluar la calidad realizando 35 preguntas cruzadas siendo representativo en un $90.8 \%$ del total de preguntas, y dando como resultado en la valoración de la calidad del servicio en 6.17 puntos sobre 10 . 
Otro modelo analizado aborda como evaluar la calidad del servicio en la actividad económica de distribución de agua y alcantarillado, y en la misma se propone evaluar la mencionada actividad realizando 35 preguntas a los usuarios con representatividad del total de 91.6 de 119. De igual forma este estudio es iniciado con los mismos parámetros que los anteriores y en su aplicación realizada se obtiene que el valor de la calidad del servicio fue de 7.36 sobre 10 puntos (Molina-Manzaba \& Wolke-Varas, 2013).

Por otra parte, para evaluar la calidad del servicio en la actividad económica de servicios de alimentación se propone un modelo de evaluación formado por 24 preguntas. En la aplicación del modelo propuesto, se obtuvieron de 399 encuestas a clientes que utilizaron el servicio una valoración de 7.19 sobre 10 puntos. La representación correspondió al 95.3\% del total de preguntas (Cedeño-Tomalá \& SánchezManzaba). Asimismo, sobre la actividad económica de la enseñanza se propone también un modelo para evaluar la calidad del servicio (PeñaSantamaría, 2013). Este modelo constituye la realización de una encuesta de 45 preguntas a los estudiantes, obteniéndose un valor de la calidad del servicio igual a 7,45 sobre 10 puntos. Cabe recalcar que las 45 preguntas representan el $97.8 \%$ del total de las 119 preguntas originales.

Otro campo también es abordado sobre la actividad económica del transporte. La evaluación de la calidad del servicio fue de 6.36 puntos sobre un total de 10 de un total de 400 encuetas realizadas (SánchezAlvarado \& Arteaga-Chica, 2013). Asimismo, en la actividad económica correspondiente a la asistencia social también se realizaron estudios como los de Jaramillo-Morales \& Cruz-Pineda (2013). En la aplicación de este modelo se obtuvo un valor de la calidad del servicio de 8,84 sobre 10 puntos. De manera similar se realizaron 400 encuetas para obtener este resultado. Se obtuvo un valor de 7.9 sobre 10 puntos de un modelo de encuesta de 42 preguntas que representan el $90.5 \%$ del total de preguntas del modelo inicial (Pluas-Merchan \& Navarro-Oyarvide, 2013).

Sobre el sector financiero fueron realizadas 560 encuestas de un modelo de encuesta de 28 preguntas, que aplicándolo, se obtuvo un valor total de la calidad del servicio de 8,73 (Álvarez-Guale, 2012).

Todos estos estudios tuvieron como variables de columnas a 7 de las 8 P's del Marketing de servicios, es decir, se ajustaron al modelo inicial, describiendo las variables: Producto, Precio, Plaza, Promoción, Personas, Perceptibilidad y Procesos. Al realizar un estudio correlacional basados 
en las variables de filas: Credibilidad, Seguridad, Acceso, Comunicación, comprensión al Cliente, tangibles, confiabilidad, Respuesta, Habilidad, Cortesía, Compromiso y Liderazgo, Planeación de Mejoras, Información y Análisis, Recursos Humanos, Administración de Procesos, Enfoque en el cliente y en el Mercado, y Resultado de Negocios, se nota que existen variables comunes entre cada actividad económica., y en cada uno de ellos se describe con énfasis la interacción personal como clave para el buen desempeño de los servicios.

Los supermercados, denominados también como autoservicios, se definen como sociedades, corporaciones o establecimientos que ofrecen servicios y productos con la finalidad de cubrir varias necesidades de consumo como la alimentación, vestimenta, insumos para el hogar, papelería, y por lo general, son concurridos diariamente por sus clientes. Estos establecimientos encaminan todos sus esfuerzos publicitarios utilizando herramientas como el marketing para poder llamar la atención de los clientes y liderar sus cuotas y segmentos de mercado, sobre los cuales existe una creciente competencia por acaparamiento de nichos (LópezJiménez \& Monroy-Antón, 2013).

En este sentido, las empresas buscan nuevas formas de captar la atención del cliente apoyándose en diferentes herramientas de estudio como lo que es el Neuromarketing (Monge-Benito \& Fernadez-Guerra, 2011). En países con alta tasa de desarrollo se han realizado varios estudios para entender la conciencia del consumidor y establecer de alguna manera la forma de prevalecer dentro del subconsciente de los consumidores. De algunos de estos estudios se colige que existe una influencia muy relévate de la calidad percibida que cambian las actitudes de los consumidores (Calvo-Porral, Martínez-Fernández, \& Juanatey-Boga, 2014).

En Ecuador existen varias cadenas de supermercados con gran cuota del mercado y posicionadas en la mente de los consumidores entre ellas se encuentran: Megamaxi-Supermaxi, Mi Comisariato, Almacenes TIA y Akí-GranAkí. Cada uno de ellos con estrategias de marketing dirigidas a los diferentes segmentos de mercado y que generan un fuerte nivel de competencia por conseguir la mayor cantidad de clientes.

Una de la pocas empresas dedicadas a la calificación de marcas y de calidad, la realiza la corporación Ekos (EKOS, 2013), mostrando un Índice Nacional de Satisfacción al Cliente (INSC) por modalidad de contratación del servicio, nombre que fue cambiado a Índice EKOS de Satisfacción al Cliente (IESC) en el año 2014 (EKOS, 2014). 


\section{Materiales y método}

El objetivo general de la presente investigación es determinar el efecto que tiene la interacción personal basada en la calidad del servicio en correlación con la decisión de compra por parte de los prospectivos consumidores de los supermercados de la ciudad de Guayaquil. De esta manera se plantean los siguientes objetivos específicos: (i) Evidenciar la relación existente entre la interacción personal basada en la calidad del servicio con el proceso de compra de los consumidores de los comisariatos de la ciudad de Guayaquil; y (ii) identificar la relación existente entre la interacción personal basada en la calidad del servicio y el comportamiento de compra en los consumidores de los comisariatos de la ciudad de Guayaquil.

En base a este contexto, como hipótesis de partida ( $\mathrm{Hg})$ se plantea que la interacción personal basada en la calidad del servicio influye en la decisión de compra por parte de los consumidores de los supermercados de la ciudad de Guayaquil, de la que surgen dos hipótesis específicas: (H1) existe relación entre la interacción personal basada en la calidad del servicio con el proceso de compra de los consumidores de los comisariatos de la ciudad de Guayaquil; y (H2) la interacción personal basada en la calidad del servicio influye en el comportamiento de compra en los consumidores de los comisariatos de la ciudad de Guayaquil.

Para cumplir con los objetivos señalados ut supra se aplicó un análisis de tipo correlacional-explicativo, debido a que se presume un vínculo existente entre las variables de estudio (Carrasco Díaz, 2013) y conllevará a determinar la existencia y el grado de relación positiva o negativa que se tenga entre la interacción personal basada en la calidad del servicio y la decisión de compra de los consumidores de los supermercados de la ciudad de Guayaquil.

Por su parte, el instrumento de recolección de datos se basó en la técnica del cuestionario, donde cada una de las treinta preguntas se valoran en una escala de Likert del 1 al 5, donde 1 se valora la percepción del encuestado como "totalmente en desacuerdo", mientras que la valoración 5 se especifica como "totalmente de acuerdo". Para la validación del cuestionario y de las preguntas contestadas por los clientes se utilizó el coeficiente de Alfa de Cronbach para el análisis de confiabilidad (Carrasco Díaz, 2013). Todas las preguntas que se formularon fueron realizadas de acuerdo a la yuxtaposición de las variables "Interacción personal" y "decisión de compra", tal y como se detalla en la tabla 1. 
Tabla 1. Matriz de operacionalización de las variables del estudio

\begin{tabular}{|c|c|c|}
\hline Variable & Dimensión & Indicadores \\
\hline \multirow{3}{*}{$\begin{array}{l}\text { Interacción } \\
\text { Personal } \\
\text { basada como } \\
\text { componente } \\
\text { de la calidad } \\
\text { del servicio }\end{array}$} & $\begin{array}{l}\text { Capacidad de respuesta de } \\
\text { los empleados de los super- } \\
\text { mercados }\end{array}$ & $\begin{array}{l}\text {-Disponibilidad de los colaborado- } \\
\text { res de los supermercados }\end{array}$ \\
\hline & \multirow{2}{*}{$\begin{array}{l}\text { Seguridad del establecimiento } \\
\text { y de de los empleados }\end{array}$} & -Transmisión de confianza \\
\hline & & -Habilidad de ayudar \\
\hline \multirow{8}{*}{$\begin{array}{l}\text { Decisión de } \\
\text { Compra de los } \\
\text { consumidores }\end{array}$} & \multirow{4}{*}{$\begin{array}{l}\text { De los consumidores: Análisis } \\
\text { del Proceso de compra }\end{array}$} & -Reconocimiento de la necesidad \\
\hline & & $\begin{array}{l}\text {-Complejidad de Búsqueda de } \\
\text { productos }\end{array}$ \\
\hline & & $\begin{array}{l}\text {-Evaluación del producto antes de } \\
\text { realizar la compra }\end{array}$ \\
\hline & & -El análisis de la Post-compra \\
\hline & \multirow{4}{*}{$\begin{array}{l}\text { De los consumidores: Com- } \\
\text { portamiento de compra }\end{array}$} & Complejo \\
\hline & & Disonancia \\
\hline & & Variedad \\
\hline & & Compras habituales \\
\hline
\end{tabular}

Fuente: Elaboración propia

Por su parte, la población del estudio está conformada por los todos aquellos clientes de los distintos supermercados de la ciudad de Guayaquil, escogidas de las cuatro cadenas más importantes según volumen de ventas. En este sentido, hasta enero de 2017 se contabiliza la existencia de 55 autoservicios.

A estos grupos que fungen como objetos muestrales se aplicará el instrumento de recopilación de datos a los consumidores que hayan realizado alguna adquisición de productos en los establecimientos antes mencionados inmediatamente después de haber realizado la compra. En la ciudad de Guayaquil existe un universo de 1252550 personas económicamente activas como compradores actuales y potenciales que escogen o podrían escoger alguno de estos centros de acopio de productos. Para determinar el tamaño de la muestra, se referirá a la siguiente fórmula: 


$$
n=\frac{Z_{\alpha / 2}^{2} N P Q}{e^{2}(N-1)+Z_{\alpha / 2}^{2} P Q}
$$

Donde el valor de $n$ se denota como el tamaño de la muestra, que es el valor que se desea obtener. Con el fin de determinar la probabilidad o porción de objetos o individuos con la características de estudio la variable $\mathrm{P}$, y para esta investigación se utilizará el valor de $\mathrm{P}=0.5$, ya que es el valor máximo de varianza por desconocimiento y no haberse realizado un estudio preliminar exploratorio. Por su parte Q se refiere a la probabilidad o proporción de objetos o individuos que no tienen las características de estudio, que sería para este estudio igual a 0.5.

Continuando con la explicación de la obtención de la muestra, e se refiere al error permitido para los resultados, que en esta investigación se permitirá margen de $+/-3 \%$. $\alpha$ es el valor de significancia o el complemento del nivel de confianza, que en el caso de estudio se plantea en 5\%, mientras que $z \alpha / 2=1.96$ para el nivel de confianza $95 \%$, según la tabla de distribución normal.

En la ciudad de Guayaquil, que es la ciudad con mayor movimiento comercial y población de Ecuador, existen 55 supermercados hasta enero de 2017, dato considerado como la población total o universo como objetos de estudio, de (1), con $\mathrm{N}=55$ :

$$
n_{s}=\frac{(1.96)^{2}(55)(0.5)(.5)}{(0.03)^{2}(55-1)+(1.96)^{2}(0.5)(0.5)}=52.35 \cong 53
$$

Donde $\mathrm{n}_{\mathrm{s}}$ es la cantidad de supermercados que serán espacio muestrales, siendo igual a 53.Tal como se ha apuntado ut supra, existe un total de 1'252.550 personas económicamente activas en la ciudad de Guayaquil, y todos ellos, que son compradores y posiblemente sean potenciales compradores de los supermercados, por lo que es necesario obtener el total de clientes a encuestar, de (1) y con N=1'252,550, $\mathrm{n}_{\mathrm{c}}$, sería:

$$
n_{s}=\frac{(1.96)^{2}(55)(0.5)(.5)}{(0.03)^{2}(55-1)+(1.96)^{2}(0.5)(0.5)}=52.35 \cong 53
$$

Así, el tamaño de la muestra efectiva queda determinado por $\mathrm{n}_{\mathrm{c}}=1067$ clientes. 
Todas las hipótesis planteadas en esta investigación son propuestas por los investigadores y se asumen la existencia de la relación entre variables y dimensiones propuestas en la matriz de operacionalización de variables, de modo que la hipótesis nula da lugar a la ausencia de correlación entre estas variables y dimensiones. Todas y cada una de las hipótesis serán contrastada mediante la siguiente regla: La hipótesis nula se acepta si valor-p de la significancia bilateral es $>$ que el nivel de significancia , que para este estudio es igual al 5\% (Walpole, Raymond, Myers, \& Keying, 2012). El valor-p se calcula de manera inmediata con en el software estadístico Statistical Package for the Social Sciences más conocido como IBM-SPSS. Además se calculará el Coeficiente de Correlación Tau_b de Kendall, ya que es necesario utilizar un coeficiente de correlación que mida la relación existente en variables de carácter ordinal. El coeficiente de correlación de Kendall es pertinente utilizarlo para este tipo de investigaciones valoradas en la escala de Likert (Badii, Guillen, Lugo Serrato, \& Aguilar Garnica, 2014).

\section{Resultados}

Para tener información confiable de las preguntas contestadas por los clientes que realizaron una compra en los supermercados, es necesario realizar en primera instancia el análisis de fiabilidad del instrumento de recopilación de información. Para esta investigación se utilizó coeficiente de Alfa de Cronbach, con el objeto de medir la fiabilidad del cuestionario. A través de la herramienta estadística SPSS se realizó el análisis de fiabilidad de las 30 preguntas planteadas, dando como resultado un coeficiente de 0.878 , por lo que se demuestra la confiabilidad del instrumento.

Acerca del objetivo general planteado de determinar el efecto que tiene la interacción personal basada en la calidad del servicio hacia la decisión de compra por parte de los consumidores de los supermercados de la ciudad de Guayaquil, se planteó las hipótesis de partida $\left(\mathrm{H}_{0}\right)$ que la interacción personal basada en la calidad del servicio no influye en la decisión de compra por parte de los consumidores de los supermercados de la ciudad de Guayaquil.

Por esta razón, se debe de contrastar la siguiente prueba de hipótesis $\left(\mathrm{H}_{\mathrm{g}}\right)$ La interacción personal basada en la calidad del servicio influye en la decisión de compra por parte de los consumidores de los supermercados de la ciudad de Guayaquil. Siendo la hipótesis nula la inexistencia de relación alguna, denotada como: $\rho=0$, y la hipótesis de investigación en donde 
se expresa la relación entre la interacción personal y la decisión de compra, denotada como $\rho \neq 0$. Simplificando, las pruebas de hipótesis quedarían:

$\mathrm{H}_{0}: \rho=0$

$\mathrm{H}_{\mathrm{g}}: \rho \neq 0$

Para contrastar la hipótesis planteada, es necesario tener una idea descriptiva del comportamiento porcentual de los datos, revisando la figura 1 se puede observar que los porcentajes de la interacción personal tienen a subir, a excepción del último descriptor. Si analizamos la decisión de compra, los datos tienen una tendencia de subida. Esto podría tener en alguna concordancia de datos entre estas dos variables.z

\section{Figura 1. Percepción de la interacción personal y la decisión de compra}

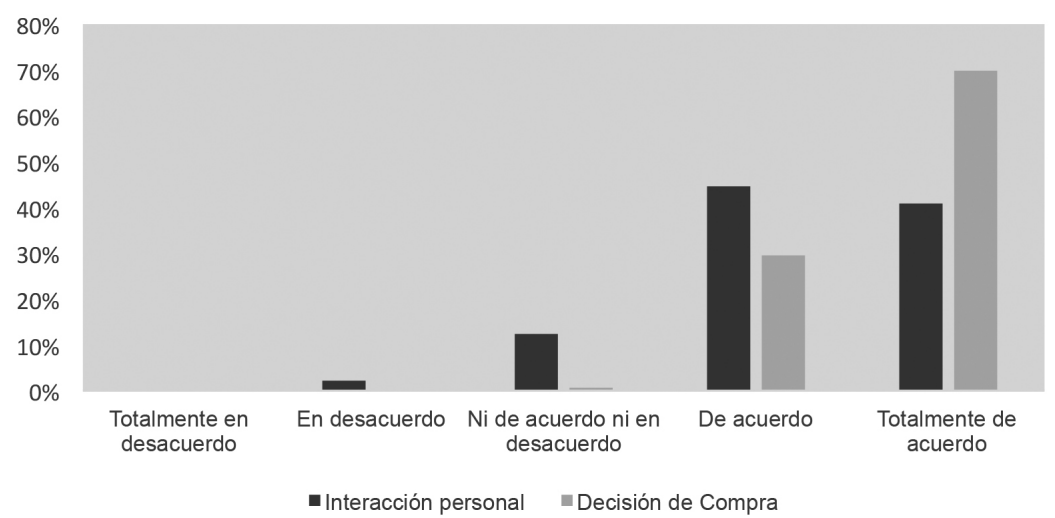

Fuente: Elaboración propia

Una vez analizado el comportamiento de datos, es necesario revisar en la tabla de correlación de Tau b de Kendall de la hipótesis general descrita en la tabla 2. Es menester observar que el valor del coeficiente de correlación es de 0.409 , por lo que se puede especular en una relación media entre correlación entre interacción personal basada como componente de la calidad del servicio y decisión de compra de los consumidores. Para la contratación de hipótesis es necesario referirnos a la metodología propuesta en esta investigación. De lo que se muestra en la tabla 3 , el valor valor-p de la significancia bilateral es igual a cero, por lo tanto menor al valor de significancia $\alpha=0.05$, y ante este contexto, se rechaza la hipótesis nula, es decir, que la interacción personal basada en la calidad 
del servicio que se percibe en las cadenas de los comisariatos en la ciudad de Guayaquil influye en la decisión de compra de sus consumidores.

Tabla 2. Correlación de Tau b de Kendall hipótesis general

\begin{tabular}{|l|l|l|}
\hline \multicolumn{2}{|c|}{} & Decisión de Compra \\
\hline \multirow{2}{*}{$\begin{array}{l}\text { Interacción } \\
\text { Personal }\end{array}$} & Coeficiente de correlación & $0.409^{* *}$ \\
\cline { 2 - 3 } & valor-p de la significancia bilateral & 0 \\
\hline \multirow{2}{*}{. La correlación es significativa en el nivel 0.01 (bilateral). } \\
\hline
\end{tabular}

Fuente: Elaboración propia

Del objetivo específico diseñado en la presente investigación, el cual trata de evidenciar la relación o el efecto existente entre la interacción personal de los empleados basada en la calidad del servicio con el proceso de compra de los clientes de los supermercados, se diseñó la siguiente hipótesis de investigación: $\mathrm{H}_{0}$ : No existe relación entre la interacción personal basada en la calidad del servicio con el proceso de compra de los consumidores de los comisariatos de la ciudad de Guayaquil. $\mathrm{H}_{1}$ : Existe relación entre la interacción personal basada en la calidad del servicio con el proceso de compra de los consumidores de los comisariatos de la ciudad de Guayaquil.

De manera similar, la hipótesis nula asegura la inexistencia de relación alguna, denotada como: $\rho=0$ para el planteamiento de esta hipótesis, y la hipótesis de investigación se enuncia la relación existente entre la interacción personal y el proceso de compra de los consumidores de los supermercados, mostrada como $\rho \neq 0$. Aclarando de forma resumida quedaría:

$\mathrm{H}_{0}: \rho=0$

$\mathrm{H}_{1}: \rho \neq 0$

Los datos graficados en la figura 2 describen de forma porcentual que el proceso de compra también tiende a subir con la interacción personal. Esto indicaría una posible relación de ambas variables. 


\section{Figura 2. Percepción de la interacción personal y el proceso de compra}

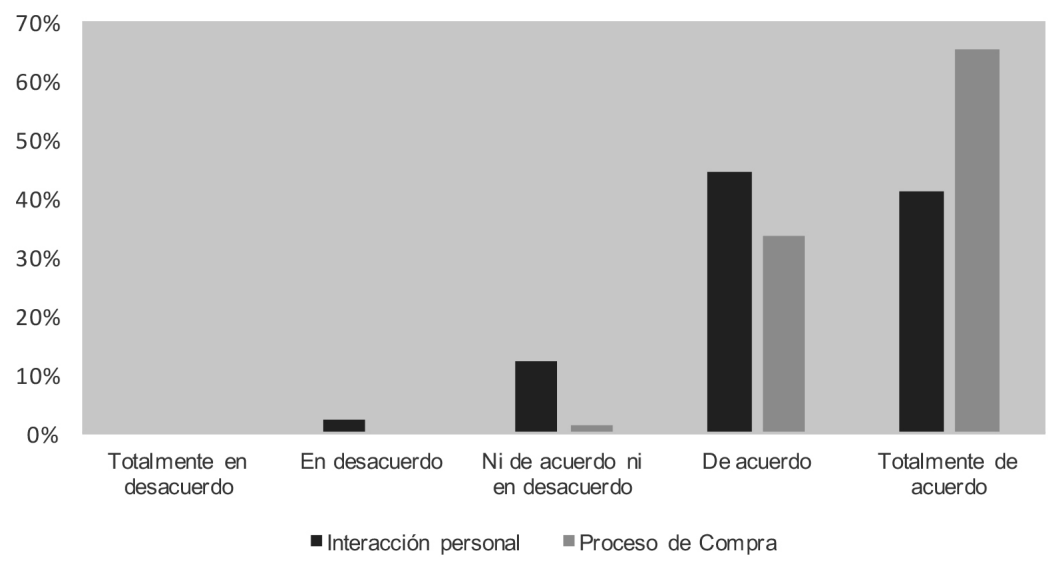

Fuente: Elaboración propia

En la tabla 3 se muestra que el coeficiente de correlación es de 0.364 , por lo que se entendería que existe en una correlación baja entre la interacción personal por parte de empleados y clientes con el proceso de compra de los consumidores. Analizando el valor de significancia bilateral, podemos constatar que ese valor es inferior al $5 \%$, por lo que se debe rechazar la hipótesis nula, es decir existe relación entre la interacción personal que perciben los clientes basada en la calidad del servicio con el proceso de compra de esos mismos comisariatos.

Tabla 3. Correlación de Tau b de Kendall hipótesis específica 1

\begin{tabular}{|l|l|l|}
\hline \multirow{2}{*}{$\begin{array}{l}\text { Interacción } \\
\text { Personal }\end{array}$} & Coeficiente de correlación & \multicolumn{1}{|c|}{ Proceso de Compra } \\
\cline { 2 - 3 } & valor-p de la significancia bilateral & 0 \\
\hline \multirow{2}{*}{$*$. La correlación es significativa en el nivel 0.01 (bilateral). } \\
\hline
\end{tabular}

Fuente: Elaboración propia

En este trabajo de investigación se propone también identificar la relación existente entre la interacción personal basada en la calidad del servicio percibida por los clientes de los supermercados de la ciudad de Guayaquil, con su comportamiento de compra de esos mismos 
consumidores. De forma similar y analizada en la comprobación de las hipótesis, se plantean las siguientes: $\mathrm{H}_{0}$ : La interacción personal basada en la calidad del servicio no influye en el comportamiento de compra en los consumidores de los comisariatos de la ciudad de Guayaquil. $\mathrm{H}_{2}$ : La interacción personal basada en la calidad del servicio influye en el comportamiento de compra en los consumidores de los comisariatos de la ciudad de Guayaquil. Resumiendo se contrasta la hipótesis:

$$
\begin{aligned}
& \mathrm{H}_{0}: \rho=0 \\
& \mathrm{H}_{2}: \rho \neq 0
\end{aligned}
$$

Observando la figura 3 se puede constatar que la interacción personal y el comportamiento de compra tienden a subir porcentualmente a medida de la calificación de percepción valorada por los clientes encuestados.

\section{Figura 3. Percepción de la interacción personal y el comportamiento de compra}

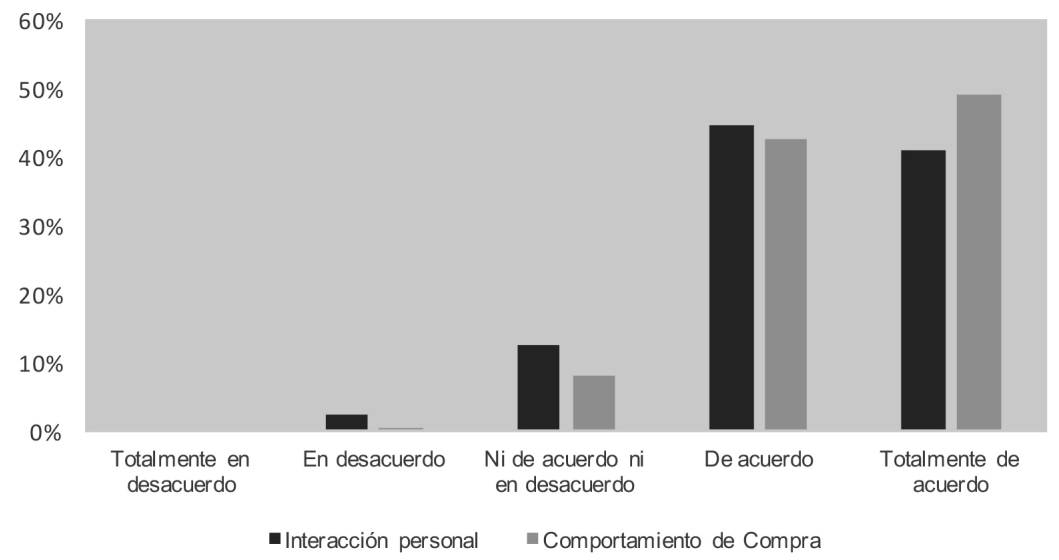

Fuente: Elaboración propia

Para contrastar la hipótesis, se determinó que el valor de la significancia bilateral fue inferior a 0.05 , por lo que no se debe aprobar la hipótesis nula, es decir, la interacción personal basada en la calidad del servicio influye en el comportamiento de compra en los consumidores de los comisariatos de la ciudad de Guayaquil, con un coeficiente de correlación de Tau b de Kendall de 0.379 . 


\section{Discusión y conclusiones}

Al determinar el efecto que tiene la interacción personal basada en la calidad del servicio hacia la decisión de compra por parte de los consumidores de los supermercados de la ciudad de Guayaquil por medio de una prueba de hipótesis contrastada mediante el coeficiente de correlación de Tau b de Kendall, se concluye con un nivel de significación del $5 \%$ de que la relación es positiva y media, debido a que el valor de la significancia bilateral fue menor a 0.05 y el coeficiente de correlación igual a 0.409. Este resultado es el más relevante de la investigación debido a que se demuestra de manera estadística que la interacción entre empleados y consumidores pueden influir en la decisión de compra de los clientes, e inclusive estudios han demostrado que se involucran el comportamiento del consumidor en el proceso de compra. (Rivas \& Ildefonso-Grande, 2013).

Se comprobó además la relación existente entre la interacción personal basada en la calidad del servicio con el proceso de compra de los consumidores de los comisariatos de la ciudad de Guayaquil, de igual manera a través de una prueba de hipótesis contrastada mediante el coeficiente de correlación de Tau b de Kendall, dicho coeficiente fue igual a 0.364 . La hipótesis se contrastó con un valor p de significancia menor a 0.05 . Se nota la existencia de una relación positiva y baja entre la interacción personal y el proceso de compra. El proceso de compra de los consumidores basados en el reconocimiento de la necesidad, e tipo de búsqueda de productos, la evaluación de compra y la post compra que realizan los clientes muestran un proceso complejo al realizar la compra (Kotler, 2002). Este proceso involucra también el efecto en el comportamiento complejo variado enfocado en varios factores (Manero \& Caraballo, 2009).

Justificada la relación entre la interacción personal basada en la calidad del servicio y el comportamiento de compra en los consumidores de los comisariatos en la ciudad de Guayaquil mediante una prueba de hipótesis contrastada usando el coeficiente de correlación de Tau b de Kendall, se notó que el nivel observado de significancia fue menor al $5 \%$, y con un coeficiente de correlación de 0.379 . Por esta razón se puede inferir que su influencia es positiva y baja. Se puede sugerir que en la actualidad la interacción personal influye de manera baja en el comportamiento de compra de los consumidores, que incluso es muy diferente de manera comparativa a aquellos consumidores que lo hacen sin tener 
una interacción personal con los empleados, como por ejemplo, los consumidores que realizan compras por medio del comercio electrónico (Arce-Urriza \& Cebollada-Calvo, 2011), y varia la compra con la sensación experimentada (Bernat-López \& Pinto-Ruiz , 2001).

Siendo la interacción personal uno de varios componentes de la calidad del servicio, se debe realizar investigaciones en otros factores involucrados como la evidencia física, la fiabilidad y las políticas de calidad para determinar algún tipo de diagrama o ecuación estructural que pueda visualizar el efecto que tiene los componentes de la calidad del servicio en la decisión de compra de los consumidores. Se pueden realizar modelos comparativos con otras actividades económicas basada en los servicios.

\section{Referencias}

Alvarado-Contreras, M. A., \& Arteaga-Mendoza, G. M. (2013). Creación y aplicación de un modelo de evaluación de la calidad del servicio orientado a la reparación de vehículos automotores y motocicletas en la ciudad de Guayaquil, aplicada a 6 vulcanizadoras de la Parroquia Ximena. Guayaquil. (Tesis de Grado, Universidad Politécnica Salesiana). (https://goo.gl/5HqXDI) (2017-03-13).

Álvarez-Guale, R. J. (2011). Estudio de correlación de las variables significativas analizadas en el censo económico 2010. Retos, 1(2), 19-37.

Álvarez-Guale, R. J. (2012). Modelo de evaluación de la calidad para instituciones financieras obtenidas por medio de un análisis de correspondencia y de clúster. Retos, 2(3), 69-94.

Arce-Urriza, M., \& Cebollada-Calvo, J. J. (2011). A comparison of consumer behavior in online and offline channels: Price sensitivity, brand loyalty, and product characteristics effect. CEDE, 102-111.

Badii, M. H., Guillen, A., Lugo Serrato, O. P., \& Aguilar Garnica, J. J. (2014). Correlación No-Paramétrica y su aplicación en la investigaciones científica. Daena, 2(9), 31-40.

Bernat-López, \& Pinto-Ruiz . (2001). La esencia del marketing. Barcelona: Ediciones UPC.

Calvo Porral, C., Martínez Fernández, V. A., \& Juanatey Boga, O. (2014). Credibilidad de los medios de comunicación: análisis de la prensa diaria desde el comportamiento del consumidor. Profesional de la Informacion, 23(3), 300-310.

Campoverde-Guerra, K. A., \& Pérez-Beltrán, K. I. (2013). Creación y aplicación de un modelo de evaluación de la calidad del servicio de distribución y venta de combustibles en tres gasolineras de la parroquia Tarqui, según corresponda a la actividad económica 'venta al por menor de combustibles para automotores. (Tesis de Grado, Universidad Politécnica Salesiana). (https://goo.gl/HhmBNp) (2017-03-12).

Carrasco-Díaz, S. (2013). Metodología de la Investigación científica. Lima: San Marcos.

Cedeño-Tomalá, M. M., \& Sánchez-Manzaba, M. T. (s.f.). Creación y Aplicación de 
un modelo de evaluación de la calidad del servicio orientado a 3 centros comerciales según correspondan a la actividad económica 'servicios de comida' en la ciudad de Guayaquil. (Tesis de Grado, Universidad Politécnica Salesiana). (https://goo.gl/U2552u) (2017-03-12).

EKOS (2013). Obtenido de http://www.ekosnegocios.com

(02 de Enero de 2014). Recuperado el 14 de Enero de 2014, http://www.ekosnegocios.com

Jaramillo-Morales, E. G., \& Cruz-Pineda, S. P. (2013). Creación y aplicación de un modelo de evaluación de la calidad del servicio orientado a 5 instituciones privadas según correspondan a la actividad económica 'Asistencia social' en la ciudad de Guayaquil. (Tesis de Grado, Universidad Politécnica Salesiana). (https://goo.gl/cCLCRf) (2017-03-13).

Kotler , P. (2002). Dirección de Marketing. México: Pearson Educación de México S.A.

López-Jiménez, D., \& Monroy-Antón, A. (2013). Quality e-commerce: Business commitments assumed for the consumer's benefit. Innovar, 23(47), 41-52.

Manero, C. B., \& Caraballo, N. M. (2009). Determining Factors of Consumer Varied Behaviour in the Store Set of Purchase. Revista Europea de Dirección y Economía de la Empresa, 18(3) 99-114.

Molina-Manzaba, J. D., \& Wolke-Varas, C. A. (2013). Creación y aplicación de un modelo de evaluación de la calidad del servicio orientado a la parroquia Tarqui según corresponda a la actividad económica Distribución de agua y alcantarillado en la ciudad de Guayaquil. (Tesis de Grado, Universidad Politécnica Salesiana). (https://goo.gl/MUF93x) (2017-03-13).

Monge-Benito, S., \& Fernández-Guerra, V. (2011). Neuromarketing: Tecnologías, Mercado y Retos. Pensar la publicidad, 5(2), 19-42.

Peña-Santamaría, M. S. (2013). Creación y aplicación de un modelo de evaluación de la calidad del servicio orientado a 5 colegios privados según corresponda la actividad económica 'enseñanza, en la ciudad de Guayaquil. (Tesis de Grado, Universidad Politécnica Salesiana). (https://goo.gl/YLWD4m) (2017-03-13).

Pluas-Merchan, I. S., \& Navarro-Oyarvide, S. L. (2013). Creación y aplicación de un modelo de evaluación de la calidad del servicio orientado a 5 instituciones públicas según corresponda a la actividad económica: 'actividades de atención humana' en la ciudad de Guayaquil. (Tesis de Grado, Universidad Politécnica Salesiana). (https://goo.gl/7CLoJG) (2017-03-12).

Rivas, X. A., \& Ildefonso-Grande, E. (2013). Comportamiento del consumidor. Madrid: ESIC.

Sánchez-Alvarado, O. A., \& Arteaga-Chica, H. V. (2013). Creación y aplicación de un modelo de evaluación de la calidad del servicio orientado al transporte ambulatorio privado según correspondan a la actividad económica 'transporte' aplicada a los taxis que operan en la parroquia Ximena de la ciudad de Guayaquil. (Tesis de Grado, Universidad Politécnica Salesiana). (https://goo.gl/ VJ7wlj) (2017-03-12).

Walpole, R., Raymond, M., Myers, S., \& Keying, Y. (2012). Probabilidad y Estadística para Ingeniería y Ciencias. México: Pearson. 